\title{
Coronary vasomotor tone during static and dynamic exercise
}

\author{
O. M. Hess, A. Bortone, K. Eid, J. E. Gage, H. Nonogi, J. Grimm and \\ H. P. Krayenbuehl \\ Department of Internal Medicine, Medical Policlinic, Cardiology, University Hospital, Zurich, \\ Switzerland
}

KEY WORDS: Coronary vasomotion, quantitative coronary arteriography, isometric and dynamic exercise, coronary sinus flow, normal and stenotic coronary arteries, coronary artery disease, endothelium-derived relaxing factor.

Coronary vasomotion is an important determinant of myocardial perfusion in patients with angina pectoris, and it influences not only normal but also stenotic coronary arteries. The ability of a stenotic coronary artery to change its size is dependent on the presence of a normal musculo elastic wall segment within the stenosis (i.e., eccentric stenosis). Coronary vasoconstriction of normal and stenotic coronary arteries has been reported by Brown and coworkers (Circulation 1984; 70: 18-24) during isometric exercise.

The effect of dynamic exericse on coronary vasomotion was evaluated in one group of 13 patients with ischaemia-like symptoms and normal coronary arteries (group 1) and in a second group of 12 patients with coronary artery disease with exercise-induced angina pectoris (group 2). Luminal area of a normal and a stenotic vessel segment was determined by biplane quantitative coronary arteriography at rest, during supine bicycle exercise and $5 \mathrm{~min}$ after administration of $1.6 \mathrm{mg}$ sublingual nitroglycerin. Coronary sinus blood flow was measured in group 1 at rest and after $0.5 \mathrm{mg} \mathrm{kg}{ }^{-1}$ intravenous dipyridamole using coronary sinus thermodilution. Coronary flow reserve was calculated from coronary sinus flow after dipyridamole divided by coronary sinus flow at rest.

In group 1, coronary vasodilation of the large (i.e., proximal) and the small (i.e., distal) coronary arteries was observed during exercise in seven patients (subgroup A). However, in the remaining six patients (subgroup B) coronary vasoconstriction of the small arteries $(-24 \%, \mathrm{P}<0.001)$ was found during exercise, whereas the large vessels showed coronary vasodilation $(+26 \%, \mathrm{P}<0.001)$. Coronary flow reserve was significantly $(\mathrm{P}<0.05)$ larger in subgroup $A$ (mean 2.5 ) than in subgroup $B$ (mean 1.2) with exercise-induced vasoconstriction of the small epicardial arteries.

In group 2 vasodilation of the normal $(+23 \%, \mathrm{P}<0.001)$ and vasoconstriction of the stenotic coronary arteries ( $-29, \mathrm{P}<0.001)$ was found during supine bicycle exercise. Administration of sublingual nitroglycerin at the end of the exercise test was accompanied by coronary vasodilation of both normal ( $+40 \%, \mathrm{P}<0.001$ vs rest) and stenotic ( $+12 \%, N S$ vs rest) vessel segments.

It is concluded that isometric exercise is associated with reflex coronary vasoconstriction of the normal and stenotic vessel segments due to enhanced sympathetic stimulation. Dynamic exercise in patients with ischaemia-like symptoms and normal coronary arteries is accompanied by an abnormal dilatory response of the small coronary arteries in a subgroup of patients with reduced coronary flow reserve. Dynamic exercise in patients with coronary artery disease is, however, associated with coronary vasodilation of the normal and coronary vasoconstriction of the stenotic vessel segments. The nature of this exercise-induced vasoconstriction of stenotic coronary arteries is not clear, but might be related to endothelial dysfunction with an insufficient production of the endothelium-derived relaxing factor during exercise.

Address for correspondence: Otto M. Hess, M. D., Medical Policlinic, Cardiology, University Hospital, Raemistrasse 100, CH-8091 Zurich, Switzerland.
This study was supported by the Swiss National Science Foundation. 


\section{Introduction}

Coronary vasomotion plays an important role in the regulation of coronary perfusion at rest and during exercise ${ }^{[1-3]}$. Not only normal but also stenotic coronary arteries show coronary vasomotion at the site of the coronary stenosis since approximately $70 \%$ of all coronary stenoses have a normal musculo-elastic wall segment within the stenosis ${ }^{\mid 4-5]}$. Previous studies have reported that during isometric exercise not only normal but also stenotic coronary arteries show coronary vasoconstriction ${ }^{|6|}$, probably due to enhanced sympathetic stimulation. Pretreatment with intracoronary nitroglycerin prevented vasoconstriction of both normal and stenotic coronary arteries, probably due to the direct vasodilating effect of nitroglycerin on the smooth vasculature of the coronary arteries. The purpose of the present study was (1) to evaluate the effect of dynamic exercise on coronary vasomotion in patients with ischaemia-like symptoms and normal coronary arteries, and (2) to study the effect of dynamic exercise on coronary vasomotion in patients with coronary artery disease with exercise-induced angina pectoris.

\section{Patients and methods}

25 patients were included in the present study. 13 patients with normal coronary arteries constituted group 1 and 12 patients with coronary artery disease group 2 . Mean age was 48 years in group 1 and 53 years in group 2. A history of angina pectoris and/or ST-segment depression in the upright bicycle exercise test was present in all patients of both groups. Coronary arteriography revealed normal coronary arteries in all group 1 patients, and one-vessel disease in one patient, two-vessel disease in two and three-vessel disease in nine patients of group 2.

\section{QUANTTTATIVE CORONARY ARTERIOGRAPHY}

Biplane coronary arteriography was performed after an interval of at least $10 \mathrm{~min}$ after the last diagnostic coronary angiogram. Baseline coronary arteriography was carried out after the patient's feet had been attached to a bicycle ergometer (Siemens-Elema AG, model 380B). Repeat biplane coronary arteriography, with concurrent aortic and pulmonary artery pressure recordings, was carried out at the end of each exercise level. Exercise was begun at $50-75 \mathrm{~W}$ and increased every $2 \mathrm{~min}$ in increments of 25-50 W and was terminated because of anginal pain, fatigue or ST-segment depression of more than $0.2 \mathrm{mV}$. Immediately after the exercise test $1.6 \mathrm{mg}$ sublingual nitroglycerin was administered and, $5 \mathrm{~min}$ later, biplane coronary arteriography was repeated.

Quantitative evaluation of biplane coronary angiograms was carried out in a blinded fashion. Tracings were made manually from the simultaneous right and left anterior oblique projection during diastasis or end-diastole. Each vessel segment was analysed four to six times and the results were averaged to reduce sampling error. A section of the catheter of known dimensions was traced as a scaling factor. The tracings were digitized and analysed on a PDP 11/34 computer $^{[3]}$. The luminal area of the proximal and distal left anterior descending and the proximal and distal left circumflex coronary artery were analysed in group 1 and the luminal area of a normal and a stenotic vessel segment, either of the left anterior descending or left circumflex coronary artery, was evaluated in group 2. Interobserver variability $(n=22)$ was $9.3 \%$ of the mean vessel area for monoplane and $7.9 \%$ for biplane measurements ${ }^{[3]}$.

\section{CORONARY SINUS BLOOD FLOW}

Flow was measured by the coronary sinus thermodilution technique ${ }^{[7]}$. Ten patients of group 1 were restudied 7-20 days (mean 10 days) after coronary arteriography on an ambulatory basis. A coronary sinus thermodilution catheter was introduced from the right femoral vein into the coronary sinus and $50 \mathrm{ml}$ saline was infused over $1 \mathrm{~min}$ to determine coronary sinus outflow according to the technique of Ganz and coworkers ${ }^{[7]}$. The first measurement was carried out at rest and the second after intravenous infusion of $0.5 \mathrm{mg} \mathrm{kg}^{-1}$ dipyridamole over $15 \mathrm{~min}$. Coronary flow ratio (i.e., coronary flow reserve) was calculated as the coronary sinus flow after dipyridamole divided by the coronary sinus flow at rest. The coronary resistance ratio was obtained by dividing the coronary resistance at rest by the coronary resistance after dipyridamole.

\section{PATIENT SUBGROUPS}

Patients with normal coronary arteries and ischaemia-like symptoms were divided into two 
subgroups according to the behaviour of the small coronary arteries during exercise: Patients with vasodilation during exercise were allocated to subgroup $\mathrm{A}(n=7)$ and patients with vasoconstriction of the small epicardial arteries during exercise to subgroup $B(n=6)$.

\section{STATISTICS}

Comparisons of haemodynamic and angiographic data in response to a first and a second exercise level and to sublingual nitroglycerin were carried out by two-way analysis of variance for repeated measurements. Comparisons between two groups or subgroups were performed by the unpaired Student $t$-test. In Figs 1-3 mean values \pm 1 standard error are reported.

\section{Results}

CORONARY LUMINAL AREA AT REST AND DURING EXERCISE

1. Patients with ischaemia-like symptoms and normal coronary arteries (Fig. 1).
Coronary vasodilation of the proximal and distal coronary arteries was found in all seven patients of subgroup $A$. The proximal vessels of the left anterior descending and left circumflex coronary artery increased by $21 \%(P<0.001)$ and the distal vessels by $45 \% \quad(P<0.001)$ during exercise. After sublingual administration of nitroglycerin, there was a further increase in luminal area of the proximal $(+46 \%, P<0.001)$ and distal $(+99 \%, P<0.001)$ coronary vessels. In contrast to subgroup $A$, there was coronary vasoconstriction of the distal coronary vessels $(-24 \%, P<0.001)$ during exercise in subgroup B. Nevertheless, the proximal coronary arteries showed, as in subgroup $A$, coronary vasodilation $(+26 \%, \quad P<0.001)$ during exercise. After sublingual administration of nitroglycerin there was an increase in luminal area of both distal $(+44 \%, \quad P<0.001)$ and proximal $(+47 \%$, $P<0.001)$ coronary arteries in subgroup $B$. Coronary flow reserve was significantly $(P<$ $0.05)$ reduced in subgroup $B(1.2 \pm 0.3 ; n=5)$ compared with subgroup A $(2 \cdot 5 \pm 1 \cdot 0 ; n=5)$. Parallel to coronary flow reserve, coronary resistance ratio was significantly $(P<0.05)$
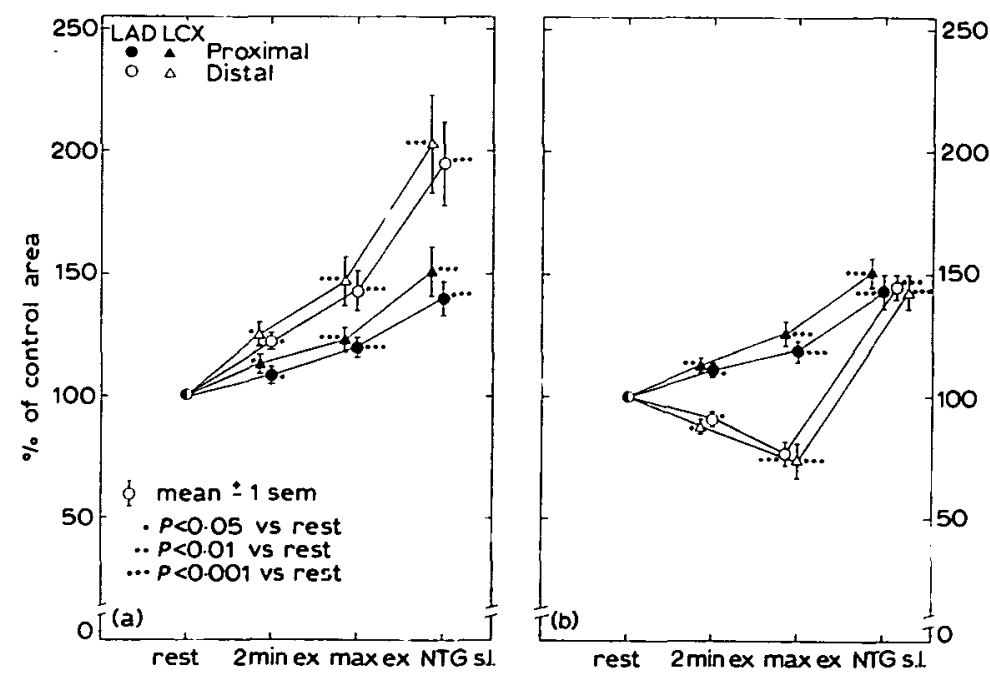

Figure 1 Response of normal coronary arteries to dynamic exercise in patients with ischaemia-like symptoms and normal coronary arteries (group 1). Proximal and distal vessel segments of the left anterior descending (LAD) and left circumflex (LCX) coronary artery were analysed at rest, after $2 \mathrm{~min}$ exercise (ex) and during maximal ex as well as 5 min after $1.6 \mathrm{mg}$ sublingual nitroglycerin (NTG s.I.). Patients $(n=7)$ who showed vasodilation of both proximal and distal coronary arteries were designated subgroup $A$ (a) and patients $(n=6)$ who showed vasoconstriction of distal but vasodilation of proximal coronary vessels were designated subgroup $B$ (b). Luminal area is given in percent of control area. Abbreviations as in Fig. 1. 
reduced in subgroup B $(1 \cdot 2 \pm 0 \cdot 3 ; n=5)$ compared with subgroup A $(2 \cdot 7 \pm 1 \cdot 2 ; n=5)$.

\section{Patients with coronary artery disease and} exercise-induced angina pectoris (Fig. 2). Coronary vasodilation of the normal coronary arteries was observed in patients with coronary artery disease $(+23 \%, P<0.001)$ during exercise. After sublingual administration of nitroglycerin, the luminal area of the normal vessel increased $(+40 \%, P<0.001)$. Stenotic coronary vessels showed coronary vasoconstriction ( $-29 \%, P<0 \cdot 001$ ) during exercise (Fig. 2) which was reversible after sublingual administration of nitroglycerin at the end of the exercise test $(+12 \%$, NS vs rest, $P<0.001$ vs exercise).

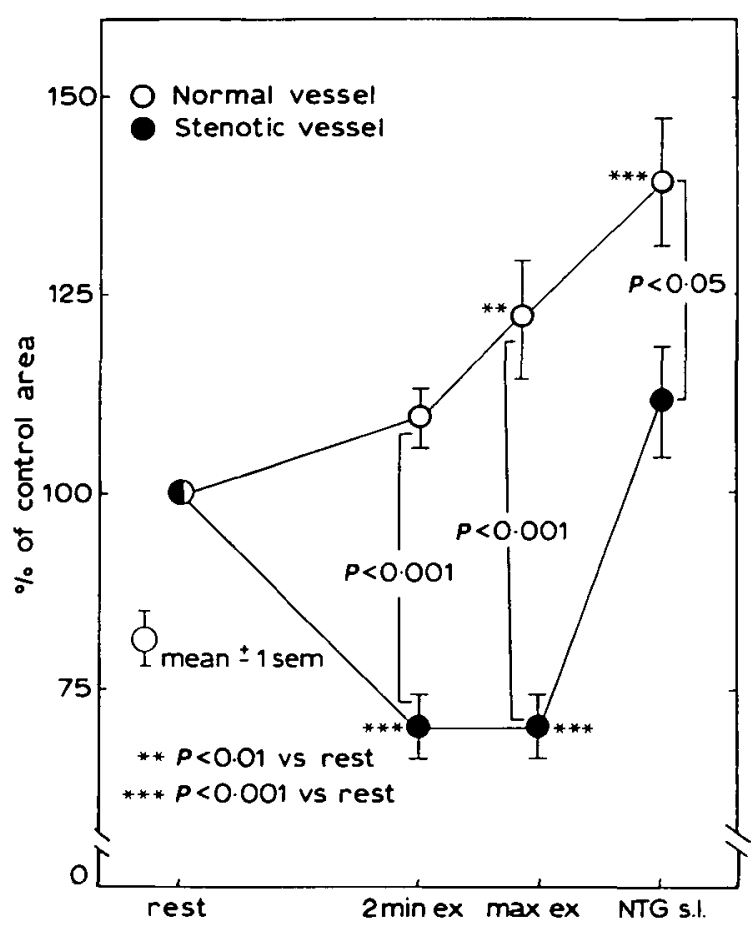

Figure 2 Responses of normal and stenotic coronary arteries to dynamic exercise in patients with coronary artery disease and exercise-induced angina pectoris. Normal vessels (open symbols) dilate during exercise to $123 \%$ of resting values, and further dilate to $140 \%$ after sublingual nitroglycerin. Stenotic vessels (closed symbols) show vasoconstriction during exercise, the stenosis narrowing to $71 \%$ of control, and vasodilation to $112 \%$ of control after sublingual nitroglycerin. Luminal area is given as $\%$ control area.
INFLUENCE OF STENOSIS SEVERITY ON CORONARY VASOMOTION

Patients with coronary artery disease were analysed separately for the influence of stenosis severity on coronary vasomotion. Three groups of coronary vessels were evaluated (Fig. 3): normal vessels $(n=7)$, stenotic vessels $(n=4)$ with mild stenosis ( $\leqslant 50 \%$ diameter stenosis) and stenotic vessels $(n=7)$ with severe stenosis ( $>50 \%$ diameter stenosis). Normal coronary arteries showed progressively coronary vasodilation during exercise and vasodilation was maximal after sublingual administration of nitroglycerin. Stenotic vessels showed, however, exercise-induced vasoconstriction which was more pronounced in severely stenotic than in mildly stenotic coronary arteries (Fig. 3). At the second exercise level, mild coronary stenoses showed only minor coronary vasoconstriction compared with the resting state $(-10 \%, \mathrm{NS})$ but severe coronary stenoses revealed significant coronary vasoconstriction during dynamic exercise $(-32 \%, P<0.001)$.

\section{Discussion}

Epicardial coronary arteries show completely different reactions to isometric or dynamic exercise in patients with coronary artery disease $^{[1,3.6]}$. This reaction to exercise is dependent on several factors and can be modified by the administration of different vasodilating substances. The purpose of the present study is to report on the effect of dynamic exercise on coronary vasomotion in patients with ischaemialike symptoms and normal coronary arteries, as well as in patients with coronary artery disease with classic exercise-induced angina pectoris.

\section{EFFECT OF ISOMETRIC EXERCISE ON CORONARY VASOMOTION}

Brown and coworkers ${ }^{|6|}$ studied the effect of isometric exercise on coronary luminal area in 11 patients with coronary artery disease. Normal and stenotic vessel segments showed coronary vasoconstriction (normal segment $-14 \%$, stenotic segment $-35 \%)$ during handgrip exercise. After pretreatment with intracoronary nitroglycerin, coronary vasoconstriction was abolished and there was coronary vasodilation of both normal $(+29 \%)$ and stenotic $(+32 \%)$ vessel segments during handgrip exercise. The authors concluded that enhanced $\alpha$-adrenergic stimula- 


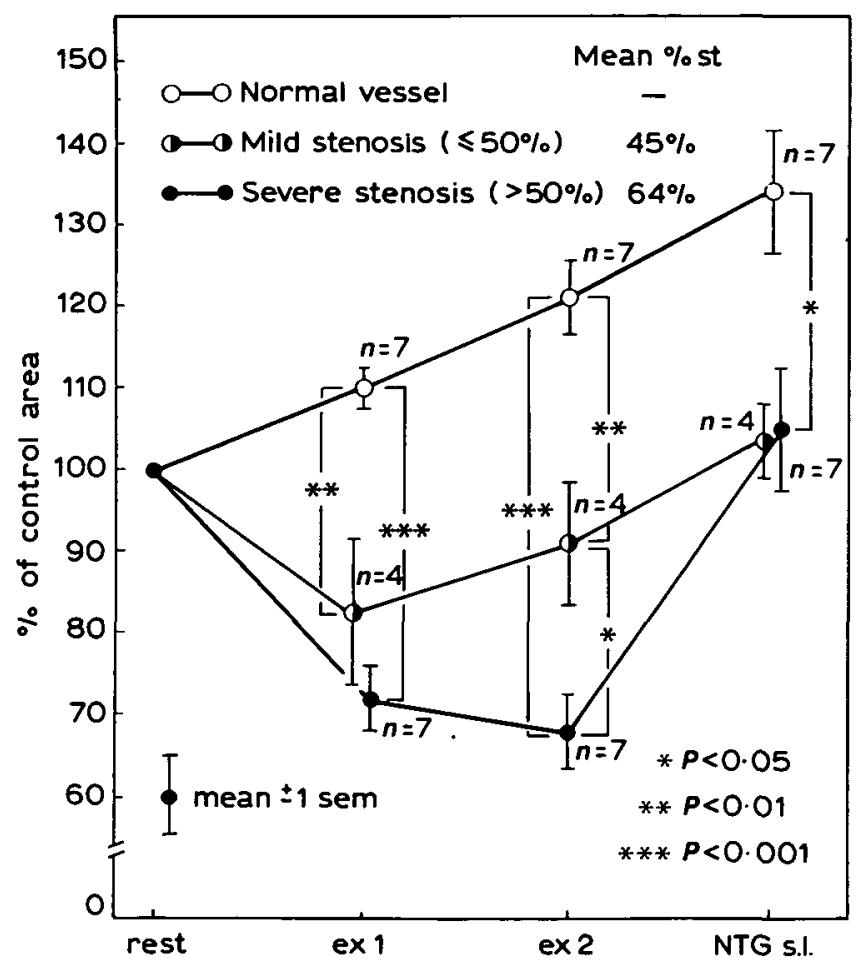

Figure 3 Influence of stenosis severity on coronary vasomotion of normal vessels (open symbols), mild stenoses (half open symbols) and severe stenoses (closed symbols) during exercise (ex1: first, and ex2: second level of exercise) and $5 \mathrm{~min}$ after administration of $1.6 \mathrm{mg}$ sublingual nitroglycerin (NTG s.l.) Normal vessels dilate progressively during exercise and after sublingual nitroglycerin, whereas stenotic vessel segments show exercise-induced vasoconstriction. Severe coronary stenoses constrict more than mild stenoses but both dilate only minimally after sublingual nitroglycerin. These data suggest that exercise-induced stenosis narrowing is dependent on the severity of coronary stenosis or, in other words, is dependent on the extent of the atherosclerotic process. Luminal area is given as \% control area; Mean \%st: Mean diameter stenosis (\%); n: number of patients.

tion with increased levels of circulating epinephrine and norepinephrine during isometric exercise was probably responsible for coronary vasoconstriction of epicardial coronary arteries in patients with coronary artery disease.

\section{EFFECT OF DYNAMIC EXERCISE ON CORONARY VASOMOTION}

Normal epicardial arteries show coronary vasodilation during dynamic exercise. Several factors are responsible for coronary vasodilation, such as a higher perfusion pressure, an increase in coronary blood flow with release of the endothelium-derived relaxing factor, an augmentation of circulating metabolites, as well as changes in neurohumoral regulation of the coronary arteries.

13 patients with normal coronary arteries and ischaemia-like symptoms were evaluated in the present study. Half were found to have abnormal coronary vasomotion of the small epicardial arteries during dynamic exercise (subgroup B). Not only was an abnormal dilatory response of the small coronary arteries observed during exercise, but also a reduced dilator capacity of the resistance vessels 
(= arterioles) was found during dipyridamole infusion. The abnormal dilatory response is likely to be due to an abnormal neurohumoral regulation of coronary vasomotor tone, as was suggested by Cannon and coworkers ${ }^{[8,9]}$ and Greenberg and coworkers ${ }^{[10]}$. Administration of sublingual nitroglycerin was associated with coronary vasodilation of the small epicardial coronary arteries, suggesting that these vessels potentially can react normally.

Dynamic exercise in patients with coronary artery disease was accompanied by coronary vasodilation of normal vessel segments but by coronary vasoconstriction of stenotic segments. This opposite effect of dynamic exercise on the coronary vasomotion of normal and stenotic coronary arteries must be explained by the following mechanisms: normal coronary arteries show vasodilation during dynamic exercise to meet the increased oxygen demands of the myocardium (see above). Stenotic coronary arteries behave somewhat paradoxically during dynamic exercise by further increasing the severity of stenosis. This phenomenon is related either to a passive collapse of the free vessel wall within the stenotic segment when coronary blood flow velocity is increased during exercise (Venturi mechanism) ${ }^{[1]}$ or to an insufficient production of endothelium-derived relaxing factor due to the atherosclerotic alterations of the vessel wall ${ }^{[3]}$. Another possible mechanism involves increased platelet aggregation with turbulent flow during exercise and with release of thromboxane $A_{2}$ and serotonin, both of which induce coronary vasoconstriction. Thus, the present results suggest that exercise-induced narrowing of coronary stenoses is due to active vasoconstriction and is an important mechanism in the production of myocardial ischaemia during dynamic exercise in patients with coronary artery disease.

\section{References}

[1] Brown BG, Bolson EL, Dodge HT. Dynamic mechanisms in human coronary stenosis. Circulation 1984: 70: 917-22.

[2] Mates RE, Gupta RL, Bell AC, Klocke FJ. Fluid dynamics of coronary artery stenosis. Circ Res 1978; 42: 152-62.

[3] Gage JE, Hess OM, Murakami T, Ritter M, Grimm J, Krayenbuehl HP. Vasoconstriction of stenotic coronary arteries during dynamic exercise in patients with classic angina pectoris: reversibility by nitroglycerin. Circulation 1986; 73: 865-76.

[4] Freudenberg $H$, Lichtlen PR. The normal wall segment in coronary stenosis-a postmortal study. Z Kardiol 1981; 70: 863-9.

[5] Saner HE, Gobel FL, Salomonowitz E, Erlien DA, Edwards JE. The disease-free wall in coronary atherosclerosis: its relation to degree of obstruction. J Am Coll Cardiol 1985; 6: 1096-9.

[6] Brown BG, Lee AB, Bolson EL, Dodge HT. Reflex constriction of significant coronary stenosis as a mechanism contributing to ischemic left ventricular dysfunction during isometric exercise. Circulation 1984; 70: 18-24.

[7] Ganz W, Tamura K, Marcus HS, Donoso $R$, Yoshida S, Swan HJC. Measurement of coronary sinus blood flow by continuous thermodilution in man. Circulation 1971; 44: 181-95.

[8] Cannon RO, Watson RM, Rosing DR, Epstein SE. Angina caused by reduced vasodilator reserve of the small coronary arteries. J Am Coll Cardiol 1983; 1: 1359-73.

[9] Cannon RO, Schenke WH, Leon MB, Rosing DR, Urqhart J, Epstein SE. Limited coronary flow reserve after dipyridamole in patients with ergonovine-induced coronary vasoconstriction. Circulation 1987; 75: 163-74.

[10] Greenberg MA, Grose RM, Neuburger N, Silverman $\mathbf{R}$, Strain JE, Cohen MV. Impaired coronary vasodilator responsiveness as a cause of lactate production during pacing-induced ischemia in patients with angina pectoris and normal coronary arteries. J Am Coll Cardiol 1987; 9: 743-51. 